\title{
Epithelial-Mesenchymal Expression Phenotype of Primary Melanoma and Matched Metastases and Relationship with Overall Survival
}

\author{
SHAOFENG YAN ${ }^{1}$, BRITT M. HOLDERNESS ${ }^{2}$, ZHONGZE LI $^{3}$, GREGORY D. SEIDEL ${ }^{1}$, \\ JIANG GUI $^{4}$, JAN L. FISHER ${ }^{5}$ and MARC S. ERNSTOFF ${ }^{6}$ \\ Departments of ${ }^{1}$ Pathology and Laboratory Medicine, and \\ ${ }^{2}$ Hematology/Oncology, Dartmouth Hitchcock Medical Center, Lebanon, NH, U.S.A.; \\ ${ }^{3}$ Biostatistics Shared Resource and ${ }^{5}$ Department of Medicine, Norris Cotton Cancer Center, and \\ ${ }^{4}$ Department of Biomedical Data Science, Geisel School of Medicine at Dartmouth, Lebanon, NH, U.S.A.; \\ ${ }^{6}$ Department of Medicine, Roswell Park Cancer Institute, Buffalo, NY, U.S.A.
}

\begin{abstract}
E-Cadherin and $N$-cadherin are important components of epithelial-mesenchymal transition (EMT). The majority of studies on EMT in melanoma have been performed with cultured cell lines or pooled melanoma samples. The goal of our study was to evaluate the expression of E-cadherin and $N$-cadherin in matched tissue samples from primary and metastatic sites of melanoma and to determine the correlation with survival outcome. We analyzed tissues from 42 melanoma primary lesions and their corresponding metastases, as well as 53 benign nevi, for expression levels of E-cadherin and $N$ cadherin using immunohistochemical methods. There were heterogenous expression patterns of $E$ - and $N$-cadherin in both primary and metastatic melanomas. Overall, metastatic tumor showed a decrease in E-cadherin expression and an increase in $N$-cadherin expression compared to the primary tumor, although the difference did not reach statistical significance ( $p=0.24$ and 0.28 respectively). A switch of membranous expression from $\mathrm{E}$-cadherin to $\mathrm{N}$-cadherin from primary to metastatic melanoma was seen in eight patients (19\%). Aberrant E-cadherin expression (defined as negative to weak membranous E-cadherin or positive nuclear E-cadherin expression) was more frequently observed in metastatic than in primary melanomas $(p=0.03)$. Multivariate analysis showed
\end{abstract}

This article is freely accessible online.

Correspondence to: Dr. Marc S. Ernstoff, Department of Medicine, Roswell Park Cancer Institute, Elm \& Carlton Streets, Buffalo, NY 14263, U.S.A. Tel: +1 7168454101, Fax: +1 7168453423, e-mail: marc.ernstoff@RoswellPark.org

Key Words: Epithelial-mesenchymal transition, E-cadherin, $\mathrm{N}$-cadherin, melanoma, metastasis. that absence of $N$-cadherin expression in primary melanomas and the presence of aberrant E-cadherin expression in primary melanomas and metastatic melanomas was associated with a significantly worse overall survival. Our data support the importance of E-cadherin and $N$-cadherin proteins in melanoma progression and patient survival.

Melanoma is one of the deadliest types of cancer, with even shallow primary lesions able to progress from early invasion to metastasis and ultimately causing death. The ability at the time of diagnosis to identify tumor characteristics that help predict propensity for metastasis would be useful in guiding the discussions about prognosis, as well as selecting patients who may benefit the most from systemic therapy. In addition, understanding the process by which metastases develop could potentially identify targets for therapeutic interventions.

Epithelial-mesenchymal transition (EMT), which was initially described in the context of embryonic development, is a means by which a cell changes morphologically and functionally from an epithelial to a mesenchymal phenotype. This change alters adhesive properties between it and adjacent cells necessary for the migration of the cell to distant sites (1). Thus EMT is involved in the initial steps of developing metastatic potential, when tumor cells escape from the microenvironment via loss of cellular adhesion and gain of motility. Following access to the circulatory or lymphatic system, tumor cells migrate to a new location, often in a visceral organ and establish metastases (2). Although melanocytes are derived from neural crest cells and are not epithelial in origin, EMT is a well-documented phenomenon contributing to the metastatic potential of malignant melanocytes $(3,4)$.

Cadherins, or calcium-dependent adhesion molecules, are transmembrane glycoproteins involved in cellular adhesion, which in skin is adhesion of melanocytes and keratinocytes. 
In vitro studies have shown that among other factors, downregulation of membrane expression of epithelial, or E-cadherin, and increase in neural, or $\mathrm{N}$-cadherin, are involved in the ability of a melanoma cell to migrate out of its nascent environment (5-8). One of the important early steps in melanoma development includes disruption of E-cadherin-mediated adhesive interaction between melanocytes and keratincoytes, accompanied by increased expression of $\mathrm{N}$-cadherin, which facilitates proliferation and invasion of melanoma cells. Expression of $\mathrm{N}$ - and E-cadherins are regulated, in part, by ubiquitin carboxylterminal hydrolase $(C Y L D)$ gene and the zinc finger protein encoded by the snail family transcriptional repressor 1 (SNAII) gene, respectively (9).

The role of cadherins in EMT has been studied in multiple tumor types. A decrease in expression of E-cadherin has been observed in oral dysplasia and squamous cell carcinoma when compared to normal controls (10), and in early-stage primary tongue cancer it has been noted to portend later development of metastases after initial glossectomy (11). Breast cancer cells have been shown to have increased metastasis when they exhibit low expression of E-cadherin and high expression of N-cadherin (12).

Studies involving analysis of melanocytic tumor tissue from primary lesions and metastases support the role of Ecadherin down-regulation in metastatic potential (11-14). These studies compared pooled primary lesions to metastases, or benign melanocytic lesions to melanomas (1316), supporting the concept that down-regulation of Ecadherin is one aspect in a complicated process that allows a melanoma cell to escape from its site of origin in the skin epidermis. If $\mathrm{E}$ - and $\mathrm{N}$-cadherin were strongly associated with melanoma metastatic potential, we hypothesize that the switch would be evident in paired primary and metastatic samples from the same patient. In order to confirm EMT in melanoma, we undertook what we believe to be the first reported evaluation of matched tissue samples from primary and metastatic sites of melanoma to confirm the presence of the $\mathrm{E}$ to $\mathrm{N}$-cadherin switch and to determine if expression profiles are associated with survival. We also examined the expression patterns of E-cadherin and $\mathrm{N}$-cadherin in 53 benign melanocytic nevi using a tissue microarray as control.

\section{Materials and Methods}

Case selection. The tumor cases were selected from formalin-fixed, paraffin-embedded tissue blocks obtained from the Department of Pathology and Laboratory Medicine at Dartmouth-Hitchcock Medical Center. The tumor cohort characteristics are presented in Table I. We reviewed the charts of 3075 patients whose melanoma tissue was archived at the Dartmouth-Hitchcock Medical Center tissue bank between 1990-2008, and identified 63 individuals with matched tissue samples from both a primary lesion and a metastatic site. Forty two out of the 63 patients had a sufficient quantity of tissue to allow immunohistochemical analysis. This study was approved by the Dartmouth College Committee for the Protection of Human Subjects/Institutional Review Board (CPHS\# 23388).

Immunohistochemistry. Immunohistochemical stains were performed on $4-\mu \mathrm{m}$ paraffin sections with the following primary antibodies: E-cadherin (clone 36, dilution 1:400; BD Transduction Laboratories, San Jose,CA, USA) and N-cadherin (clone 3B9, dilution 1:150; Invitrogen Life Technologies, Waltham, MA, USA). Immunohistochemical stains were performed using Leica Bond Automated stainer (Leica Biosystem, Buffalo Grove, IL, USA), Bond Epitope Retrieval 1 (Leica Biosystem), and Bond polymer Red refine detection kit (BD Biosciences, San Diego, CA, USA) according to the manufacturers' instructions. Omission of primary antibody was performed as a negative control. In addition, for $\mathrm{N}$ cadherin, sections of myocardial tissue were used as a positive control and sections of bladder mucosa were used as a negative control. Normal skin epidermis was used as a positive control for E-cadherin immunostaining and dermis was used as a negative control. Pairing of tissue samples was blinded to the pathologists and slides of all immunohistochemical studies were reviewed by two independent dermatopathologists (SY and GS).

Evaluation of immunohistochemical staining. Immunohistochemical staining by these two antibodies were measured by semiquantitative assessment of positive staining in subcellular location of tumor cells as described previously (16). Only membranous and nuclear stains were evaluated. Scoring included both the percentage of positive staining ( 0 point $<1 \%, 1$ point $=1-10 \%, 2$ points $=11$ $50 \% ; 3$ points $=51-80 \%$; and 4 points $\geq 80 \%$ ) and the intensity of staining ( 1 point=weak, 2 points=moderate, 3 point $=$ strong). Staining in fewer than $1 \%$ cells was considered negative regardless of staining intensity. An overall score was calculated for each tissue sample by adding the percentage score to the intensity score, resulting totals of $0-1$ points were considered negative and received an overall score of 0,2-3 points was considered weak and received an overall score of $1,4-5$ points was considered moderate and received an overall score of 2, and 6-7 points was considered strong and received an overall score of 3. E-Cadherin was considered to have normal membranous expression when the overall score was 2 or 3 , and aberrant expression when the overall nuclear score $>0$, or with a membrane score of 0 or 1 . Cadherin expression was considered to switch from $\mathrm{E}$ - to $\mathrm{N}$-cadherin when a patient had both a decrease of membranous E-cadherin and increase of membranous $\mathrm{N}$-cadherin expression in the metastatic tissue compared to the primary melanoma tumor.

Statistical analysis. SAS 9.4 (SAS Institute Inc., Cary, NC, USA) software was used for data analyses. Wilcoxon signed-rank tests and binomial proportion tests were used to compare the E-cadherin and $\mathrm{N}$-cadherin expression patterns in primary melanomas and metastases. The McNemar agreement test was applied to test whether distribution of aberrant E-cadherin in primary and metastatic sites agreed. All tests were two-sided with significance level set to 0.05 .

Overall survival data were analyzed using the Kaplan-Meier method, and estimated hazard ratios of prognostic factors were derived from univariate and multivariate Cox's proportional hazard models. Mantel-Cox log-rank score was used to assess statistical significance of survival differences. 


\section{Results}

Our cohort of 42 individuals included 14 females and 28 males, ages 23-89 years (median age 63 years), with disease ranging from stage I to IV at initial presentation. All patients had a biopsy-proven metastasis, either present at the time of diagnosis, or having developed subsequently. There were 10 superficial spreading melanomas, nine nodular melanomas, four acral lentiginous melanomas and 19 melanomas not otherwise specified. Clinical and histological features of the patients and their tumors are described in Table I. The median tumor Breslow thickness was $3.75 \mathrm{~mm}$ (range=0.6-29 mm).

Formalin-fixed paraffin-embedded tissue from 42 primary melanomas and their paired metastatic melanoma was analyzed by immunohistochemistry for expression of Ecadherin and N-cadherin proteins (Table II and Figure 1). Moderate to strong membranous E-cadherin expression (score 2 or 3 ) was observed in $90.5 \%$ of primary melanomas and $78.6 \%$ of metastatic melanomas. Negative or weak membranous E-cadherin staining (score 0 or 1 ) was observed in $9.6 \%$ of the primary melanomas and $21.4 \%$ of the metastatic melanomas. Weak to moderate nuclear expression of E-cadherin was observed relatively infrequently in primary (19.0\%) and metastatic melanoma (28.6\%). Nuclear E-cadherin staining was absent from most melanoma cases. The majority of primary melanomas and metastatic melanomas were also negative for $\mathrm{N}$-cadherin expression (64.3\% and $59.5 \%$ respectively). Weak to moderate (score 1 or 2) membranous $\mathrm{N}$-cadherin expression was only observed in $35.7 \%$ of primary melanomas and $40.5 \%$ of metastatic melanomas. No nuclear expression of $\mathrm{N}$-cadherin was observed in primary or metastatic melanomas. Figure 1 shows $\mathrm{E}$ - and $\mathrm{N}$-cadherin staining patterns seen in three representative matched samples.

A switch of cadherin membranous expression with a decrease of membranous E-cadherin and increase of $\mathrm{N}$-cadherin membranous expression from primary to metastatic melanoma was seen in eight patients. Aberrant E-cadherin expression was defined as negative to weak membranous E-cadherin or any positive nuclear E-cadherin expression. Out of the 42 patients, 24 did not have aberrant E-cadherin in either their primary or metastatic melanomas, seven had aberrant E-cadherin in both their primary and metastatic melanomas, nine had aberrant E-cadherin expression in only their metastatic melanomas and two had aberrant E-cadherin expression in only their primary melanoma. Overall, aberrant E-cadherin expression was observed in nine $(21.4 \%)$ primary melanomas and 16 $(38.0 \%)$ metastatic melanomas. The distribution of aberrant E-cadherin in primary versus metastatic melanomas was significantly different (Table III, $p=0.03$ based on McNemar agreement test). However, we found no statistical difference of membranous E-cadherin expression, nuclear E-cadherin
Table I. Summary of tumor cases and histology.

\begin{tabular}{lc}
\hline & Cases, $\mathrm{n}$ \\
\hline Gender & \\
Male & 28 \\
Female & 14 \\
Primary site & \\
Head/neck & 6 \\
Trunk & 7 \\
Upper extremity & 15 \\
Lower extremity & 14 \\
Ulceration & \\
Present & 26 \\
Absent & 16 \\
Histological type & \\
Superficial spreading & 10 \\
Nodular & 9 \\
Acral lentiginous & 4 \\
NOS & 19 \\
Clinical stage at diagnosis & \\
I & 2 \\
IB/II & 1 \\
II & 11 \\
II/III & 4 \\
III & 21 \\
IV & 2 \\
Could not be determined & 1 \\
\hline
\end{tabular}

NOS: Not otherwise specified.

expression and membranous $\mathrm{N}$-cadherin expression when comparing primary lesions to their matched metastases (Table III, $p=0.06,0.29$ and 0.52 , respectively, based on Wilcoxon signed-rank test).

We further evaluated the membranous expression patterns of these two proteins in the 42 primary melanomas and their paired metastatic lesions (Table IV). Four co-expression patterns of membranous E-cadherin and $\mathrm{N}$-cadherin were observed. In primary melanomas, $31 \%$ of cases were positive for both markers, while $64.3 \%$ of cases were positive for E-cadherin only. Moreover, $4.8 \%$ of cases expressed $\mathrm{N}$-cadherin only and no case was negative for both markers. The distribution of coexpression in paired metastatic melanoma tissue was broadly similar as shown in Table IV.

Among patients with a change in the membranous expression of these two proteins from their primary melanomas to their paired metastatic melanomas, there were more patients with a decrease in E-cadherin expression than those with an increase in E-cadherin expression [16 patients $(61.5 \%) v s .10$ patients $(38.5 \%)]$, and there were more patients with an increase in $\mathrm{N}$-cadherin expression than those with a decrease in $\mathrm{N}$-cadherin expression [13 patients (61.9\%) vs. eight patients (38.1\%)]. However, the difference did not reach statistical significance $(p=0.24$ and 0.28 , respectively based on binomial proportion tests). We note 


\section{Case $1 \quad$ Case $2 \quad$ Case 3}

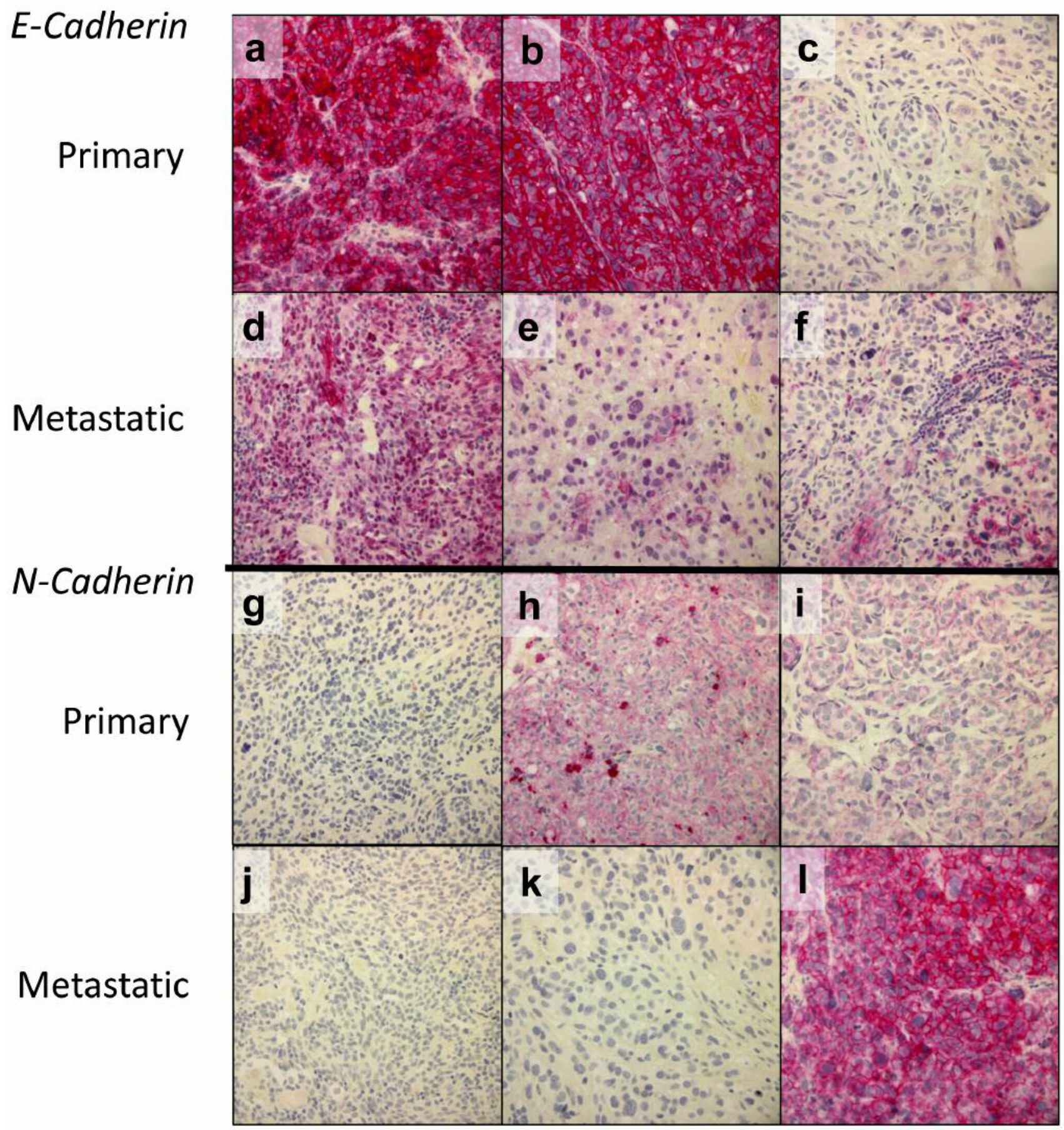

Figure 1. Representative immunohistochemical expression patterns of E-cadherin (a-f) and $N$-cadherin ( $g$-l) in primary and metastatic melanomas. Case $1(a, d, g, j)$ had strong membranous E-cadherin staining in the primary lesion (a), but loss of membranous E-cadherin staining with an increase of its nuclear staining in the matched metastatic lesion $(d) ; N$-cadherin expression was negative in both primary and metastatic lesions of this case ( $g$ and $j)$. Case $2(b, e, h, k)$ had strong membranous E-cadherin staining and moderate membranous $N$-cadherin staining in the primary lesion ( $b$ and $h$ ); membranous E-cadherin staining was weak and $N$-cadherin staining negative in its matched metastatic lesion (e and $k$ ). Case 3 $(c, f, i, l)$ had focal weak membranous E-cadherin staining and weak membranous $N$-cadherin staining in the primary lesion (c and $i) ;$ membranous $E$-cadherin staining was focal and moderate and membranous $N$-cadherin staining was strong in the corresponding metastatic lesion ( $f$ and $l$ ). Original magnification, $\times 400$. 
Table II. Cadherin expression in 42 melanoma primary lesions and their corresponding metastases.

\begin{tabular}{|c|c|c|c|c|c|}
\hline \multirow[b]{2}{*}{ Cadherin } & \multirow[b]{2}{*}{ Overall staining score } & \multicolumn{2}{|c|}{ Primary melanoma expression, n (\%) } & \multicolumn{2}{|c|}{ Metastatic melanoma expression, $\mathrm{n}(\%)$} \\
\hline & & Membranous & Nuclear & Membranous & Nuclear \\
\hline \multirow[t]{4}{*}{ E-Cadherin } & 3 & $22(52.4 \%)$ & 0 & $17(40.5 \%)$ & 0 \\
\hline & 2 & $16(38.1 \%)$ & $2(4.8 \%)$ & $16(38.1 \%)$ & $4(9.5 \%)$ \\
\hline & 1 & $2(4.8 \%)$ & $6(14.3 \%)$ & $3(7.1 \%)$ & $8(19.1 \%)$ \\
\hline & 0 & $2(4.8 \%)$ & $34(81.0 \%)$ & $6(14.3 \%)$ & $30(71.4 \%)$ \\
\hline \multirow[t]{4}{*}{ N-Cadherin } & 3 & 0 & 0 & 0 & 0 \\
\hline & 2 & $6(14.3 \%)$ & 0 & $9(21.4 \%)$ & 0 \\
\hline & 1 & $9(21.4 \%)$ & 0 & $8(19.1 \%)$ & 0 \\
\hline & 0 & $27(64.3 \%)$ & $42(100 \%)$ & $25(59.5 \%)$ & $42(100 \%)$ \\
\hline Aberrant E-cadherin expression & & \multicolumn{2}{|c|}{$9(21.4 \%)$} & \multicolumn{2}{|c|}{$16(38.1 \%)$} \\
\hline
\end{tabular}

that there were eight individuals who had a switch in cadherin expression with a decrease in the membranous E-cadherin score and an increase in the membranous $\mathrm{N}$-cadherin score when comparing the primary lesions to their matched metastatic lesions.

In order to further evaluate the association of E-cadherin and $\mathrm{N}$-cadherin expression on overall survival, a multivariate survival analysis was performed, incorporating 10 variables (Table V). There was a significant correlation between overall survival and the absence of membranous $\mathrm{N}$-cadherin expression in primary melanomas $(p=0.046)$, the presence of aberrant E-cadherin expression in primary melanomas $(p=0.030)$, and the presence of aberrant E-cadherin expression in metastatic melanomas $(p=0.048)$, as well as tumor depth $(p=0.049)$ and male gender $(p=0.004)$ (Table V).

We also examined E-cadherin and $\mathrm{N}$-cadherin expression in 53 benign nevi as controls (data not shown). The majority of benign nevi (91\%) exhibited membranous expression of E-cadherin but were negative for $\mathrm{N}$-cadherin expression, supporting the idea that high levels of E-cadherin play a role in anchoring benign melanocytic nevi in their epithelial environment.

\section{Discussion}

Cell-cell adhesion molecules have been investigated as potential factors relating to the degree of cancer invasiveness and metastasis (17). E-Cadherin is known to be a mediator of human melanocyte adhesion to keratinocytes, which, under normal circumstances keeps melanocytes from leaving their original environment in the epidermis of skin (18). Decreased expression of E-cadherin is believed to be correlated with increased ability for melanoma cell migration $(19,20)$. Kreizenbeck et al. reported a statistically significant down-regulation of E-cadherin when comparing pooled primary lesions to pooled metastatic lesions, and also suggested a correlation between improved survival and
Table III. Comparison of cadherin expression in primary versus metastatic melanomas.

\begin{tabular}{lc}
\hline Cadherin expression, primary $v s$. metastatic melanoma & $p$-Value \\
\hline Membranous E-cadherin & 0.06 \\
Nuclear E-cadherin & 0.29 \\
Aberrant E-cadherin & 0.03 \\
Membranous N-cadherin & 0.52 \\
\hline
\end{tabular}

increased expression of E-cadherin with a trend toward statistical significance. In addition, they reported that some primary lesions with increased levels of $\mathrm{N}$-cadherin were more likely to metastasize, while others which had a more well-differentiated phenotype were not (15). Massoumi et al. found that the expression of SNAIL correlated with the loss of CYLD expression and activation of the N-cadherin promoter, and was linked to tumor invasiveness and thickness in melanoma tissue (21). Loss of E-cadherin was found to be associated with proto-oncogene B-raf (BRAF) mutation and Breslow thickness greater than $1 \mathrm{~mm}$ in primary cutaneous melanoma samples from 68 patients (22).

Melanocytes normally express E-cadherin, and loss of E-cadherin is a hallmark of EMT. Loss of E-cadherin in melanoma can be associated with an increase of $\mathrm{N}$-cadherin expression (23). In the current study, more than half of the patients showed the pattern of E-cadherin-positive/ $\mathrm{N}$-cadherin-negative expression in their melanomas $(64.3 \%$ in primary melanoma and $54.8 \%$ in metastatic melanoma respectively), while about one-third of patients had positive expression of both proteins in their tumors $(31 \%$ in primary melanoma, and $31 \%$ in metastatic melanoma). Only $4.8 \%$ of primary melanomas and $9.5 \%$ of metastatic melanomas had a pattern of E-cadherin-negative/ $\mathrm{N}$-cadherin-positive expression. Overall, positive expression of $\mathrm{N}$-cadherin was 
Table IV. Co-expression patterns of E-cadherin and N-cadherin.

\begin{tabular}{|c|c|c|c|c|c|c|c|}
\hline \multirow[b]{3}{*}{ Membranous expression } & & \multicolumn{6}{|c|}{$\mathrm{N}$-Cadherin, $\mathrm{n}(\%)$} \\
\hline & & \multicolumn{3}{|c|}{ Primary melanoma } & \multicolumn{3}{|c|}{ Metastatic melanoma } \\
\hline & & + & - & Total & + & - & Total \\
\hline \multirow[t]{2}{*}{ E-Cadherin } & + & $13(31.0 \%)$ & $27(64.3 \%)$ & $40(95.2 \%)$ & $13(31 \%)$ & $23(54.8 \%)$ & $36(85.7 \%)$ \\
\hline & - & $2(4.8 \%)$ & 0 & $2(4.8 \%)$ & $4(9.5 \%)$ & $2(4.8 \%)$ & $6(14.3 \%)$ \\
\hline Total & & $15(35.7 \%)$ & $27(64.3 \%)$ & $42(100 \%)$ & $17(40.5 \%)$ & $25(59.5 \%)$ & $42(100 \%)$ \\
\hline
\end{tabular}

Table V. Multivariate analysis of the association of E-cadherin and $N$-cadherin expression on overall survival.

\begin{tabular}{llcc}
\hline Variable & & Hazard ratio (95\% CI) & $p$-Value* \\
\hline Membranous E-cadherin & Primary melanoma & $5.06(0.83-30.90)$ & 0.080 \\
& Metastatic melanoma & $3.01(0.60-15.20)$ & 0.180 \\
Membranous N-cadherin & Primary melanoma & $0.31(0.10-0.98)$ & 0.046 \\
& Metastatic melanoma & $1.79(0.57-5.64)$ & 0.320 \\
Aberrant E-cadherin expression & Primary melanoma & $3.79(1.13-12.72)$ & 0.030 \\
Switch from E-cadherin to N-cadherin & Metastatic melanoma & $4.07(1.01-16.38)$ & 0.048 \\
Tumor depth & Metastatic melanoma & $0.23(0.03-1.52)$ & 0.130 \\
Gender & Increasing & $1.09(1.00-1.19)$ & 0.049 \\
Age at diagnosis & Male vs. female & $7.59(1.90-30.40)$ & 0.004 \\
\hline
\end{tabular}

CI: Confidence interval.*By Cox model.

observed in $35.7 \%$ of primary melanomas and $40.5 \%$ of metastatic melanoma, suggesting that an inverse coupling of $\mathrm{N}$-cadherin and E-cadherin expression is not absolute.

Similar heterogenous expression patterns of E-cadherin and $\mathrm{N}$-cadherin have been described in 695 melanoma tissue cores in the literature (8), the majority of which were from metastatic lesions. In that study, $36 \%$ of cases were E-cadherin-positive/N-cadherin-negative, $23 \%$ of cases were the converse, and there were also cases in which expression of both proteins was present $(17 \%)$ or absent $(11 \%)$. Some melanomas were negative for either E-cadherin or $\mathrm{N}$-cadherin expression, suggesting a more complex control of EMT. They also measured cadherin expression in 12 melanoma cell lines. While the majority of cell lines exhibited $\mathrm{N}$-cadherin expression (58\%), only $25 \%$ of the cell lines expressed E-cadherin. The patient melanoma tissue cores exhibited more heterogenous expression patterns than did the cell lines, indicating a more complex control of EMT in the intact tumor microenvironment. In situ melanomas can express other cadherins such as dysadherin or vascular endothelial cadherin, which have been associated with aggressive behavior and poor prognosis (24). It is possible that these or other cadherins are counter-regulated in ways that extend beyond the EMT. Further studies evaluating and understanding the tumor microenvironment, cytokines that are produced, and their effects on EMT are needed.

Alonso et al. evaluated the E-cadherin to N-cadherin switch in expression levels when comparing primary lesions that later metastasized to primary lesions that did not develop metastases at follow-up, and found an association between expression of $\mathrm{N}$-cadherin and other markers of EMT (3). They reported that an increase in expression of $\mathrm{N}$-cadherin was associated with a loss of expression of cadherin 10 , which functions in a similar manner to E-cadherin, and also that induction of re-expression of E-cadherin in melanoma cells down-regulated expression of $\mathrm{N}$-cadherin in those cells. The switch of cadherin from E-cadherin to N-cadherin in melanoma was reported to be regulated by the phosphoinositide 3-kinase (PI3K) and phosphatase and tensin homolog (PTEN) pathway through the twist transcription factor (TWIST) and SNAIL (25). Data from our study support heterogeneous subsetting of melanomas based on E-cadherin and $\mathrm{N}$-cadherin expression patterns. Our data also support the observation that treatment with $\mathrm{ADH}-1$, an $\mathrm{N}$-cadherin antagonist, showed no correlation with survival in a melanoma xenograft model, although ADH-1 therapy did augment melphalan permeability into tumor cells and increased the response rate (26). The heterogenous 
expression of $\mathrm{N}$-cadherin in tumor cells may be one explanation for this. Thus, other approaches may need to be developed to enhance outcomes.

The majority of studies on melanoma EMT were performed in cultured cell lines or pooled melanoma samples, and to our knowledge, this has not been assessed in matched primary and metastatic lesions. Protein expression in cell lines may or may not reflect in vivo expression patterns. Furthermore, previously published immunohistochemical expression patterns of E-cadherin in melanoma varied from complete absence of membranous Ecadherin staining in radial growth phase melanoma, with restoration of expression in the vertical growth phase (13), to positive expression in all melanomas (14). N-Cadherin expression was only reported in a minor percentage of cases (19). In our study, positive expression of $\mathrm{N}$-cadherin was observed in $35.7 \%$ of primary melanomas and $40.5 \%$ of metastatic melanoma although one would expect more tumors to express $\mathrm{N}$-cadherin, especially the metastatic tumors. This finding suggests that regulation of EMT and metastasis in melanoma in vivo is a more complex process. Another possible explanation is that once tumor cells have established metastasis in tissue they undergo the reverse process of EMT, i.e. mesenchymal-epithelial transition to become established in the new environment (27).

Nuclear localization of E-cadherin, proposed to be linked to mutations of the $\beta$-catenin gene, has been shown in solid pseudopapillary tumors of the pancreas (28), Merkel cell carcinomas (29), and synovial sarcomas (30) using the same E-cadherin antibody (clone 36) used in our study. This aberrant expression may be manifested by negative membranous expression or positive nuclear expression of Ecadherin in tumor cells. We observed more patients with aberrant expression of E-cadherin in metastatic lesions than in their paired primary lesions, indicating an important role of loss of normal membranous expression of E-cadherin in tumor metastasis.

The prognostic significance of $\mathrm{E}$ - and $\mathrm{N}$-cadherin in melanoma are variable in the literature. Tucci et al. showed that a low E-cadherin level is prognostic of poor outcome (31). Bachamann et al. reported that the expression of $\mathrm{E}$ and $\mathrm{N}$-cadherin did not show statistical significance in predicting survival, although they found a decrease of E-cadherin expression and an increase of $\mathrm{N}$-cadherin expression from primary melanoma to metastatic melanoma (32). Kreizenbeck et al. showed that increased N-cadherin expression improved overall survival, but the significance of this disappeared when adjusted for established clinicopathological parameters (15). Multivariate analysis in our study also showed that the presence of $\mathrm{N}$-cadherin expression in the primary melanomas was associated with increased overall survival. In addition, aberrant E-cadherin expression in primary melanomas, aberrant E-cadherin expression in metastatic melanomas, tumor depth and patient gender were all associated with significantly worse overall survival, although p-values of some of these variables were borderline to the 0.05 significance level, indicating that future studies with a larger sample size are needed.

Our study, which is the first to be based on paired primary and metastatic melanoma samples, supports the importance of E-cadherin and $\mathrm{N}$-cadherin proteins in melanoma progression and metastasis, and in understanding EMT and metastasis development in melanoma. This complex process needs further characterization in the context of the tumor microenvironment, with a larger sample size for additional statistical power.

\section{Acknowledgements}

The Authors would like to thank Rebecca R. O'Meara for her excellent technical support. The immunohistochemistry study was performed at the Pathology Translational Research Laboratory, a shared core facility in the NCI-designated Norris Cotton Cancer Center.

Research reported here was supported by the Dartmouth Clinical and Translational Science Institute, under award number UL1TR001086 from the National Center for Advancing Translational Sciences (NCATS) of the National Institutes of Health (NIH). The content is solely the responsibility of the author(s) and does not necessarily represent the official views of the NIH.

\section{References}

1 Christiansen JJ and Rajasekaran AK: Reassessing epithelial to mesenchymal transition as a prerequisite for carcinoma invasion and metastasis. Cancer Res 66(17): 8319-8326, 2006.

2 Guarino M, Rubino B and Ballabio G: The role of epithelialmesenchymal transition in cancer pathology. Pathology 39(3): 305-318, 2007.

3 Alonso SR, Tracey L, Ortiz P, Pérez-Gómez B, Palacios J, Pollan M, Linares J, Serrano S, Sáez-Castillo AI, Sánchez L, Pajares R, Sánchez-Aguilera A, Artiga JM, Piris MA and Rodríguez-Peralto JL: A high-throughput study in melanoma identifies epithelialmesenchymal transition as a major determinant of metastasis. Cancer Res 67(7): 3450-3460, 2007.

4 Caramel J, Papadogeorgakis E, Hill L, Browne GJ, Richard G, Wierinckx A, Saldanha G, Osborne J, Hutchinson P, Tse G, Lachuer J, Puisieux A, Pringle JH, Ansieau S and Tulchinsky E: A switch in the expression of embryonic EMT-inducers drives the development of malignant melanoma. Cancer Cell 24(4): 466-480, 2013.

5 Hsu MY, Meier FE, Nesbit M, Hsu JY, Van Belle P, Elder DE and Herlyn M: E-cadherin expression in melanoma cells restores keratinocyte-mediated growth control and down-regulates expression of invasion-related adhesion receptors. Am J Pathol 156(5): 1515-1525, 2000.

6 Li G, Satyamoorthy K and Herlyn M: N-cadherin-mediated intercellular interactions promote survival and migration of melanoma cells. Cancer Res 61(9): 3819-3825, 2001.

7 Kim JE, Leung E, Baguley BC and Finlay GJ: Heterogeneity of expression of epithelial-mesenchymal transition markers in melanocytes and melanoma cell lines. Front Genet 4: 97, 2013. 
8 Mikesh LM, Kumar M, Erdag G, Hogan KT, Molhoek KR, Mayo MW and Slingluff CL: Evaluation of molecular markers of mesenchymal phenotype in melanoma. Melanoma Res 20(6): 485-495, 2010.

9 Barrallo-Gimeno A and Nieto MA: The SNAIL genes as inducers of cell movement and survival: implications in development and cancer. Development 132(14): 3151-3161, 2005

10 Chaw SY, Majeed AA, Dalley AJ, Chan A, Stein S and Farah CS: Epithelial to mesenchymal transition (EMT) biomarkers E-cadherin, beta-catenin, APC and Vimentin - in oral squamous cell carcinogenesis and transformation. Oral Oncol 48(10): 9971006, 2012.

11 Sakamoto K, Imanishi Y, Tomita T, Shimoda M, Kameyama K, Shibata K, Sakai N, Ozawa H, Shigetomi S, Fujii R, Fujii M and Ogawa K: Overexpression of SIP1 and down-regulation of Ecadherin predict delayed neck metastasis in stage I/II oral tongue squamous cell carcinoma after partial glossectomy. Ann Surg Oncol 19(2): 612-619, 2012.

12 Hazan RB, Phillips GR, Qiao RF, Norton L and Aaronson SA: Exogenous expression of $\mathrm{N}$-cadherin in breast cancer cells induces cell migration, invasion, and metastasis. J Cell Biol 148(4): 779-790, 2000

13 Silye R, Karayiannakis AJ, Syrigos KN, Poole S, van Noorden S, Batchelor W, Regele H, Sega W, Boesmueller H, Krausz T and Pignatelli M: E-cadherin/catenin complex in benign and malignant melanocytic lesions. J Pathol 186(4): 350-355, 1998.

14 Sanders DS, Blessing K, Hassan GA, Bruton R, Marsden JR and Jankowski J: Alterations in cadherin and catenin expression during the biological progression of melanocytic tumours. Mol Pathol 52(3): 151-157, 1999.

15 Kreizenbeck GM, Berger AJ, Subtil A, Rimm DL and Gould Rothberg BE: Prognostic significance of cadherin-based adhesion molecules in cutaneous malignant melanoma. Cancer Epidemiol Biomarkers Prev 17(4): 949-958, 2008.

16 Valencak J, Kittler H, Schmid K, Schreiber M, Raderer M, Gonzalez-Inchaurraga $\mathrm{M}$, Birner $\mathrm{P}$ and Pehamberger $\mathrm{H}$ : Prognostic relevance of hypoxia inducible factor-1alpha expression in patients with melanoma. Clin Exp Dermatol 34(8): e962-964, 2009.

17 Haass NK, Smalley KS, Li L and Herlyn M: Adhesion, migration and communication in melanocytes and melanoma. Pigment Cell Res 18(3): 150-159, 2005.

18 Tang A, Eller MS, Hara M, Yaar M, Hirohashi S and Gilchrest BA: E-Cadherin is the major mediator of human melanocyte adhesion to keratinocytes in vitro. J Cell Sci 107(Pt 4): 983-992, 1994.

19 Krengel S, Groteluschen F, Bartsch S and Tronnier M: Cadherin expression pattern in melanocytic tumors more likely depends on the melanocyte environment than on tumor cell progression. J Cutan Pathol 31(1): 1-7, 2004.

20 Seleit IA, Samaka RM, Basha MA and Bakry OA:Impact of Ecadherin expression pattern in melanocytic nevi and cutaneous malignant melanoma. Anal Quant Cytopathol Histpathol 34(4): 204-213, 2012.

21 Massoumi R, Kuphal S, Hellerbrand C, Haas B, Wild P, Spruss T, Pfeifer A, Fässler R and Bosserhoff AK: Down-regulation of CYLD expression by SNAIL promotes tumor progression in malignant melanoma. J Exp Med 206(1): 221-232, 2009.
22 Mitchell B, Leone DA, Feller JK, Yang S and Mahalingam M: BRAF and epithelial-mesenchymal transition in primary cutaneous melanoma: a role for SNAIL and E-cadherin? Hum Pathol 52: 19-27, 2016.

23 Kuphal S and Bosserhoff AK: Influence of the cytoplasmic domain of E-cadherin on endogenous $\mathrm{N}$-cadherin expression in malignant melanoma. Oncogene 25(2): 248-259, 2006.

24 Nishizawa A, Nakanishi Y, Yoshimura K, Sasajima Y, Yamazaki $\mathrm{N}$, Yamamoto A, Hanada K, Kanai $\mathrm{Y}$ and Hirohashi $\mathrm{S}$ : Clinicopathologic significance of dysadherin expression in cutaneous malignant melanoma: immunohistochemical analysis of 115 patients. Cancer 103(8): 1693-1700, 2005.

25 Hao L, Ha JR, Kuzel P, Garcia E and Persad S: Cadherin switch from $\mathrm{E}$ - to N-cadherin in melanoma progression is regulated by the PI3K/PTEN pathway through TWIST and SNAIL. Br J Dermatol 166(6): 1184-1197, 2012.

26 Turley RS, Yoshihiro T, Hiroaki T, Lidsky ME, Padussis JC, Fontanella A, Deng W, Augustine CK, Beasley GM, Davies MA, Dewhirst MW and Tyler DS: Targeting N-cadherin increases vascular permeability and differentially activates AKT in melanoma. Ann Surg 261(2): 368-377, 2015.

27 Bednarz-Knoll N, Alix-Panabieres C and Pantel K: Plasticity of disseminating cancer cells in patients with epithelial malignancies. Cancer Metastasis Rev 31(3-4): 673-687, 2012.

28 Chetty R and Serra S: Membrane loss and aberrant nuclear localization of E-cadherin are consistent features of solid pseudopapillary tumour of the pancreas. An immunohistochemical study using two antibodies recognizing different domains of the E-cadherin molecule. Histopathology 52(3): 325330, 2008.

29 Han AC, Soler AP, Tang CK, Knudsen KA and Salazar H: Nuclear localization of E-cadherin expression in Merkel cell carcinoma. Arch Pathol Lab Med 124(8): 1147-1151, 2000.

30 Izumi T, Oda Y, Hasegawa T, Nakanishi Y, Kawai A, Sonobe H, Takahira T, Kobayashi C, Yamamoto H, Tamiya S, Hiroshashi $\mathrm{S}$, Iwamoto $\mathrm{Y}$ and Tsuneyoshi M: Dysadherin expression as a significant prognostic factor and as a determinant of histologic features in synovial sarcoma: special reference to its inverse relationship with E-cadherin expression. Am J Surg Pathol 31(1): 85-94, 2007.

31 Tucci MG, Lucarini G, Brancorsini D, Zizz A, Pugnaloni A, Giacchetti A, Ricotti $G$ and Biagini G: Involvement of Ecadherin, beta-catenin, Cdc42 and CXCR4 in the progression and prognosis of cutaneous melanoma. Br J Dermatol 157(6): 1212-1216, 2007.

32 Bachmann IM, Straume O, Puntervoll HE, Kalvenes MB and Akslen LA: Importance of P-cadherin, beta-catenin, and Wnt5a/frizzled for progression of melanocytic tumors and prognosis in cutaneous melanoma. Clin Cancer Res 11(24 Pt 1): 8606-8614, 2005. 